\title{
L'adjectif dans la désignation des énergies marines renouvelables
}

\author{
Maria Francesca Bonadonna ${ }^{\mathrm{a}}$, Marianna Lisi ${ }^{\mathrm{b}}$ \\ Université de Vérone - Dipartimento di Lingue e Letterature Straniere, L.ge Porta Vittoria, 41, 37129 Verona, Italie
}

\begin{abstract}
Résumé. Cet article propose une description de l'adjectif dans le domaine des énergies marines renouvelables. Il s'inscrit dans le cadre des méthodes exploitant le système des fonctions lexicales dans la structuration des terminologies. Premièrement, un aperçu est donné sur les recherches autour de la terminologie des énergies renouvelables et sur l'adjectif. En deuxième lieu, nous menons une analyse sémantique de quelques adjectifs employés dans les unités terminologiques qui se réfèrent aux principaux types d'énergies marines renouvelables. Dans ce but, un corpus de textes publiés entre 2004 et 2015 est constitué à partir de deux genres textuels différents, le discours institutionnel et le discours scientifique, et soumis au logiciel TermoStat. Le filtrage des résultats obtenus par cet extracteur donne un échantillon de termes - ici appelés lexies spécialisées - dont la tête est représentée par énergie/énergies et qui désignent des sources énergétiques liées à l'eau. Si l'étude du sémantisme propre aux adjectifs, et de leurs relations sémantiques réciproques, fait l'objet d'une première description au moyen des fonctions lexicales, c'est l'emploi dans les lexies spécialisées qui est ensuite analysé. Cela permet de décrire leurs sens respectifs et leur positionnement sémantique à l'intérieur d'une terminologie relativement récente, dont une systématisation est souhaitable.
\end{abstract}

\begin{abstract}
L'adjectif dans la désignation des énergies marines renouvelables. This paper focuses on the adjectives of marine renewable energy and exploits a lexical functions-based approach to terminology structuring. To start with, an overview is given of research studies conducted on renewable energy terminology and on adjective. Then, we offer a semantic analysis of some adjectifs designating the main types of marine renewable energy. A corpus of texts issued between 2004 and 2015 is built on two different textual genres, namely institutional discourse and scientific discourse, and analysed through the software TermoStat. It gives a sample of terms - designated herein as lexies spécialisées whose noun head is represented by énergie/énergies and which refer to water sources of energy. First, we explore the meaning of single adjectives and their semantic relationships by encoding them with lexical functions; secondly, we consider their semantic status in specialised lexical units in order to propose a first systematisation of a recent class of terms.
\end{abstract}

\section{Introduction}

Dans cette contribution, nous nous proposons de réfléchir sur le statut de l'adjectif dans la désignation d'une nouvelle classe d'énergies renouvelables, à savoir les énergies marines renouvelables. Appelées également énergies renouvelables en mer ou, tout simplement, énergies marines, elles se réfèrent à

\footnotetext{
${ }^{a}$ Auteur de correspondance : mariafrancesca.bonadonna@univr.it

${ }^{\mathrm{b}}$ Auteur de correspondance : marianna.lisi@univr.it
} 
« l'ensemble des technologies qui permettent de produire de l'énergie, notamment de l'électricité, à partir des différentes ressources du milieu marin : la houle, les courants, les marées, le gradient de température, mais aussi le vent (éolien en mer) ${ }^{1}$. Il s'agit d'un secteur qui n'est pas sans intérêt pour la France, qui dispose d'un potentiel remarquable et qui a pour ambition d'en devenir leader mondial ${ }^{2}$.

Les énergies renouvelables suscitent l'attention dans plusieurs travaux de terminologie, axés, entre autres, sur les questions de l'harmonisation terminologique au niveau juridique (Zanola 2008), de la description lexicographique et du traitement terminographique (Giaufret - Rossi 2012; Giaufret 2013). Ces recherches portent tant sur le secteur considéré dans son ensemble que sur ses différents sous-domaines, par exemple le photovoltaïque (Zanola 2012). Nous voulons aborder le sujet avec un regard particulier sur les énergies marines renouvelables, qui, à notre connaissance, n'ont pas encore été observées. Nous entendons proposer ici quelques pistes de réflexion sur les adjectifs relationnels utilisés dans les unités terminologiques qui désignent les principaux types d'énergies de ce domaine en pleine phase de développement.

Au préalable s'imposent des considérations sur la notion d'adjectif et sur l'approche, basée sur les fonctions lexicales, que nous adoptons pour notre analyse. À propos de l'adjectif, nous rappelons que plusieurs études (Bartning 1980 ; Bosredon 1988 ; Goes 1993 ; Noailly 1999 ; Riegel 1993) ont mis en évidence la dichotomie adjectif qualificatif / adjectif relationnel, fondée sur des critères à la fois sémantiques et syntaxiques. Ces derniers sont représentés par des restrictions appliquées à l'adjectif de relation, qui refuse, en général, la construction attributive, la variation en degré, l'antéposition au nom, la coordination avec un adjectif qualificatif et l'apposition, et qui prévoit un ordre strict dans le syntagme nominal (Bosredon 1988). D'autres recherches laissent de côté la dimension syntagmatique pour privilégier la composante sémantique de l'adjectif. D'après Wierzbicka (1988), cette partie du discours se distingue du substantif principalement en raison de sa différente « semantic structure ». Dixon (1982) propose un classement sémantique des adjectifs en sept classes, qui est susceptible de s'appliquer à n'importe quelle langue : « dimension », « physical property », « colour », « human propensity », « age », « value ». Dans une récente étude sur la définition lexicographique de l'adjectif, Frassi (2014) part de la notion de fonction lexicale élaborée par la Lexicologie Explicative et Combinatoire et propose une modélisation des adjectifs correspondant à des conversions pures et à des dérivés sémantiques actanciels purs. Nous rappelons également, en ce qui concerne plus précisément l'adjectif en langue de spécialité, les études de Daille (2001) autour de l'extraction de l'adjectif relationnel en corpus, la recherche de L'Homme (2004) sur les adjectifs dérivés sémantiques dans la structuration des terminologies et celle de Maniez sur l'adjectif de relation dans le domaine médical (Maniez 2009).

Notre étude vise également l'application des principes de la Lexicologie Explicative et Combinatoire (Mel'čuk, Clas, Polguère 1995), branche lexicale de la Théorie Sens-Texte (Žolkovskij, Mel'čuk 1967 ; Mel'čuk 1974), à la terminologie. L'outil descriptif qui lui est propre, le système des fonctions lexicales, (Mel'čuk 1996) est susceptible de fournir une formalisation du sémantisme des termes à structure prédicative, ainsi qu'une représentation à large spectre de leurs relations lexicales (L'Homme 2007 ; 2012). Nous adoptons ce modèle pour la description de quelques termes, ici appelés lexies spécialisées. En premier lieu, nous proposons une analyse sémantique des adjectifs dérivés sémantiques (purs, actianciels, potentiels), indépendamment de la tête nominale. Deuxièmement, nous allons reconsidérer leurs sens respectifs, lorsqu'ils se combinent avec le vocable ENERGIE. À l'issue de cette analyse, nous allons décrire les liens lexicaux qui se tissent entre lexies spécialisées.

\section{Les lexies spécialisées dans le corpus}

Notre corpus se compose de textes de deux types, institutionnel et scientifique, ayant pour objet le domaine des énergies marines renouvelables. Dans le cadre du discours institutionnel, nous avons examiné un ensemble d'études mises en ligne entre 2013 et 2015 par le Ministère de l'Écologie, du Développement durable et de l'Énergie ; d'autres recherches à caractère purement scientifique, publiées entre 2004 et 2008, ont été consultées à partir du site du CNRS et de l'archive ouverte pluridisciplinaire HAL. Pour l'examen de ces textes, nous avons choisi d'avoir recours à l'extracteur terminologique TermoStat ${ }^{3}$. Bien que le choix du nom tête énergie soit opéré a priori - notre intérêt est 
restreint aux différents types d'énergie exploitant l'élément aquatique - le logiciel nous offre les résultats relatifs à la fréquence et à la structure morphosyntaxique des lexies spécialisées telles qu'elles apparaissent dans le corpus. Il nous permet également d'observer les contextes dans lesquels figurent les lexies spécialisées afin de mieux analyser leur sémantisme.

Après avoir soumis notre corpus au logiciel, nous avons lancé la recherche en langue française en sélectionnant le paramètre «termes complexes nominaux », alors que les «termes simples » ont été exclus de notre analyse, de manière à ne retenir que les adjectifs figurant dans des syntagmes nominaux. À partir de la liste des «candidats de regroupement» élaborée par le logiciel, nous avons effectué la sélection d'un échantillon de lexies spécialisées sur la base de deux critères, l'un morphosyntaxique, l'autre sémantique. En ce qui concerne le premier, nous ne retenons que les termes composés d'un nom et d'un ou plusieurs adjectifs. Deuxièmement, notre attention se focalise sur les termes complexes nominaux dont le substantif est représenté par énergie/énergies et dont le deuxième élément adjectival désigne des sources énergétiques liées à l'eau. Notre hypothèse est que ces adjectifs de relation indiquent une catégorie d'énergie dotée de propriétés bien déterminées. À ce propos, Bartning (1980:70) attribue le pouvoir de sous-classification à cette catégorie d'adjectifs, appelés pseudo-adjectifs ou PA : «D'une manière générale, les PA classifient et sous-catégorisent les noms, qui, à leur tour, classifient (ou instituent) un monde d'objets (les référents) ou de phénomènes présupposés réels ou existants [...]».

En adoptant ces paramètres, nous isolons dans notre corpus sept lexies spécialisées, présentées sur la base de leur fréquence : énergie marine $\left(104^{4}\right)$, énergies marines renouvelables (22), énergie hydrolienne (11), énergie houlomotrice (6), energie hydraulique (6), énergie marémotrice (6), énergie osmotique (3). Il est intéressant de remarquer que la matrice Nom Adjectif, qui caractérise ces lexies, à l'exception de la lexie énergies marines renouvelables (Nom Adjectif Adjectif), est la plus répandue même lorsque l'on se penche sur les statistiques concernant l'ensemble du corpus. En effet, elle représente $35 \%$ du total, étant suivie par les matrices suivantes : Nom Préposition Nom= 771 (19\%); Nom Nom= 419 (10\%); Nom Préposition Nom Adjectif= $338(8 \%)$; Nom PPAdj= $329(8 \%)$; Nom Adjectif Adjectif $=221(6 \%)$; Nom Adjectif PPAdj= 108 (3\%); Nom Adjectif Préposition Nom=101 (3\%); Nom Préposition Nom Préposition Nom= $101(3 \%)^{5}$. Bien que nous nous limitions, dans la présente recherche, à des cas spécifiques, ces résultats pourraient se révéler significatifs pour une étude plus ample sur l'adjectif dans la la terminologie du domaine. Le choix d'un encodage au moyen des fonctions lexicales permet vraisemblablement de "capturer», pour chaque lexie spécialisée, un nombre important de relations sémantiques, sans besoin de déployer plusieurs modèles descriptifs (L’Homme 2012 : 34).

\section{L'adjectif pour un sous-classement des énergies renouvelables}

Nous allons maintenant proposer une analyse sémantique et une modélisation des adjectifs au moyen des fonctions lexicales élaborées dans le cadre de la Théorie Sens-Texte. Né sur la formulation mathématique $f(x)=y$ et appliqué au lexique, cet outil vise une correspondance entre un argument $(x)$ et un nombre indéterminé de valeurs $(y)$, permettant ainsi de codifier les relations entre deux lexies $(f)$ : les dérivations sémantiques, des liens sémantiques de nature paradigmatique recurrents en langue ; les collocations, des affinités de combinatoire lexicale au sein d'expressions semi-idiomatiques.

En premier lieu, notre attention se focalise sur les adjectifs identifiés dans les lexies spécialisées - marin, renouvelable, hydrolien, houlomoteur, hydraulique, osmotique, marémoteur - indépendamment de leur emploi dans les syntagmes nominaux. Nous suivons l'ordre de fréquence des lexies spécialisées dans lesquelles ils sont utilisés. Pour chaque adjectif, nous nous attachons à décrire la/les composante(s) sémantique(s) et, en l'occurrence, le lien avec la base nominale ou verbale dont il est dérivé ; nous élaborons également les fonctions lexicales qui les décrivent. Deuxièmement, nous nous penchons sur ces adjectifs dans les lexies spécialisées : après avoir défini le vocable ENERGIE qui en constitue le substantif, nous examinons le lien de l'adjectif avec ENERGIE et les relations entre ces lexies spécialisées, elles aussi représentées par les fonctions lexicales. Une telle description à deux niveaux permet d'observer, dans une première phase, le ou les sens de chaque adjectif dépourvu de la tête nominale ainsi que les relations réciproques; ensuite, d'en préciser le sens à l'intérieur des lexies spécialisées dans le domaine des 
énergies marines renouvelables et d'observer dans quelle mesure ils contribuent à sous-classer les différents types d'énergie. Comme Bartning (1980: 93) l'affirme : « [...] le sens ou la définition ne ressort, à notre avis, d'une manière évidente que lorsqu'il leur donne un contexte avec le nom tête. Ceci confirme qu'il faut prendre en considération le nom tête lors d'une classification des adjectifs ».

Pour reconstuire le sens tant des adjectifs que des lexies spécialisées, nous nous appuyons également sur

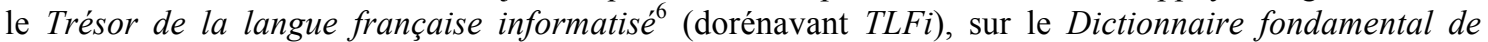
l'environnement (dorénavant DicoEnviro) ${ }^{7}$ et sur le Dictionnaire Environnement ${ }^{8}$ (dorénavant DicoE).

\subsection{Les adjectifs marin, renouvelable, hydrolien, houlomoteur, hydraulique, osmotique, marémoteur}

Le premier adjectif sur lequel nous nous concentrons est marin. Dans le DicoE l'adjectif n'est pas enregistré, alors que le DicoEnviro présente deux résultats, dont le premier concerne le domaine des énergies renouvelables. Cependant, aucune définition de l'adjectif n'est ici fournie. Comme nous pouvons l'observer dans le $T L F i$, il s'agit d'un adjectif polysémique car il peut avoir trois sens différents :

A. 'de mer, de la mer' : 1. En parlant de ce qui y vit, s'y produit, en provient ou lui appartient ; 2 . En parlant d'un être mythique ; En parlant de ce qui se trouve au bord de la mer; En parlant de ce qui a pour sujet la mer ;

B. 'qui se rapporte à la navigation sur mer' ;

C. vx. (En parlant d'une embarcation) 'Qui tient bien la mer, qui est apte à naviguer'.

Il s'agit d'un adjectif dénominal dérivé de la base non autonome mar-, du nom mer, au moyen du suffixe -in, ce dernier étant commun aux adjectifs relationnels (Daille 2001 : 152). Marin pourrait être décrit comme une conversion pure $\mathrm{A}_{0}$ à partir de la base nominale mer :

$$
\mathrm{A}_{0}(\mathrm{mer})=\text { marin }
$$

À propos de renouvelable, seul le TLFi donne une définition de l'adjectif : 'qui peut être renouvelé'. Celle-ci peut comprendre les sens suivants :

- $\quad$ A. 'Qui peut être remplacé; dont certains éléments peuvent être remplacés';

- $\quad$ B. 1. 'Qui peut être répété, qui peut se produire à nouveau';2. 'Qui peut être reconduit, dont on peut prolonger la validité'.

Renouvelable est dérivé du verbe renouveler qui présente une structure prédicative de type ' $\mathrm{X}$ renouvelle Y'. À propos de la structure prédicative, L'Homme affirme que la terminologie est plus à l'aise avec les unités non-prédicatives qu'avec les structures prédicatives telles que les verbes et les adjectifs (2012: $32)^{9}$. Nous pouvons décrire l'adjectif par une conversion pure de type $\mathrm{A}_{2}$ :

$$
\mathrm{A}_{2}(\text { renouveler })=\text { renouvelable }
$$

En ce qui concerne l'adjectif hydrolien, il n'est enregistré ni dans le TLFi ni dans le DicoE. Le DicoEnviro recense cet adjectif dans le domaine des énergies renouvelables mais il n'offre aucune définition. Nous constatons que cet adjectif est composé de hydro-, du grec v̋ $\delta \omega \rho$ 'eau', et du suffixe adjectival -ien, qui, comme Daille le rappelle (2001: 152), caractérise la formation des adjectifs relationnels. D'un point de vue sémantique, si nous admettons que ce suffixe peut exprimer 'l'idée d'origine, d'appartenance ou d'agent' (TLFi), hydrolien pourrait signifier 'ayant origine dans l'eau, appartenant à l'eau, causé par l'eau'. La consultation du corpus permet de mieux préciser le sens de l'adjectif car le substantif masculin hydrolien est également attesté avec le sens de type d'énergie renouvelable, par exemple dans la phrase «l'ADEME [...] s'est intéressée à plusieurs sources d'énergies marines comme l'éolien en mer, le houlomoteur ou encore l'hydrolien ». Nous pourrions alors décrire l'adjectif en tant que conversion pure du substantif hydrolien: 


$$
\mathrm{A}_{0}\left(\text { hydrolien }_{\mathrm{N}}\right)=\text { hydrolien }_{\mathrm{A}}
$$

De surcroît, le susbtantif féminin hydrolienne est repérable dans le corpus dans des lexies spécialisées telles qu'hydrolienne à membrane et hydrolienne fluviale. Le DicoE définit hydrolienne en tant que 'turbine immergée qui utilise l'énergie des courants marins, et parfois fluviaux, pour produire de l'électricité. À l'instar des éoliennes qui captent l'énergie cinétique des vents dans l'air, les hydroliennes exploitent les fortes vitesses des courants marins'. D'après le DicoEnviro, l'hydrolienne est utilisée par l'agent-homme pour intervenir sur le patient-courant et produire le résultat-électricité. Par un procédé analogique avec éolienne / éolien, l'adjectif hydrolien semblerait avoir aussi le sens de 'ayant origine dans une hydrolienne' (Loc) ou 'causé par une hydrolienne' (Instr) :

$$
\begin{aligned}
& \mathrm{A}_{31 \text { loc }}\left(\text { hydrolienne }_{\mathrm{N}}\right)=\text { hydrolien }_{\mathrm{A}} \\
& \mathrm{A}_{3 \text { instr }}\left(\text { hydrolienne }_{\mathrm{N}}\right)=\text { hydrolien }_{\mathrm{A}}
\end{aligned}
$$

Passons maintenant à l'adjectif houlomoteur, qui n'est pas attesté dans le TLFi. Il est fort visible qu'il relève d'un procédé de composition à partir des substantifs houle et moteur. La reconstruction de son sémantisme nous donne comme paraphrase 'qui utilise, qui se sert de la force de la houle'. Si nous abordons la définition de houle, 'mouvement ondulatoire de grande amplitude qui agite la surface de la mer ${ }^{10}$, nous remarquons que la composante sémantique du 'mouvement' figure déjà dans le sens du premier radical. Or, sa juxtaposition avec -moteur, en tant qu'élément de composition, défini comme 'qui concerne ou utilise la force motrice de' $(T L F i)$, renforce l'idée d' 'exploitation du mouvement de la houle' sans pourtant créer de redondance. Étant donné que le DicoEnviro n'enregistre aucune définition pour houlomoteur, le critère de compositionnalité sémantique nous semble la seule voie d'accès au sens de l'adjectif. C'est sur cette base que nous lui attribuons une fonction lexicale plus complexe :

$$
\mathrm{A}_{0 \text { instr }}(\text { force motrice })=\text { houlomoteur }
$$

Quant à l'adjectif hydraulique, son emploi caractérise autant la langue générale qu'un contexte de spécialité. Le TLFi le définit comme 'qui est mû par l'eau' ou 'qui est fourni par le mouvement de l'eau'; le DicoEnviro situe l'adjectif à l'intérieur du domaine des énergies renouvelables, en cooccurrence privilégiée avec le nom énergie ${ }^{11}$. Nous rappelons aussi que l'adjectif hydraulique, du fait du suffixe ique (Daille 2001 : 4), témoigne d'une régularité morphologique.

$$
\mathrm{A}_{0 \mathrm{instr}}(\mathrm{eau})=\text { hydraulique }
$$

Considérons, ensuite, l'adjectif osmotique qui signifie 'qui est relatif à l'osmose' selon le TLFi, mais qui est absent du DicoEnviro ; par l'ajout du suffixe -ique, il dérive du substantif de matrice anglaise osmose, à savoir un 'phénomène caractérisé par le passage de la solution la moins concentrée vers la solution la plus concentrée' (TLFi). Par ricochet, l'adjectif osmotique peut être représenté comme :

$$
\mathrm{A}_{0}(\text { osmose })=\text { osmotique }
$$

Nous allons conclure cette première partie de l'analyse par l'adjectif marémoteur. Il renvoie au même cas de composition que celui décrit dans houlomoteur mais, à sa différence, il est décrit, dans le TLFi, comme 'qui concerne ou utilise la force motrice des marées'. Dans le DicoEnviro, son appartenance au domaine des énergies renouvelables est confirmée et, de même, sa relation syntagmatique avec énergie.

$$
\mathrm{A}_{0 \text { instr }}(\text { force motrice })=\text { marémotrice }
$$

À partir de cette analyse, il résulte trois catégories d'adjectifs : des conversions pures $\mathrm{A}_{0}$ (marin, hydraulique, osmotique), deux dérivés actanciels $\mathrm{A}_{2}$ et $\mathrm{A}_{3}$ (renouvelable, hydrolien) et des compositions 
décrites par des fonctions lexicales complexes (houlomoteur, marémoteur). L'examen des adjectifs indépendamment du nom tête et du contexte n'est, pourtant, pas satisfaisant. D'abord, parce que la polysémie de certains adjectifs - c'est le cas de marin - ne permet pas de restreindre le sens pertinent dans le domaine des énergies renouvelables. De surcroît, parce que tous les adjectifs ne sont pas définis dans les dictionnaires consultés. Il s'avère, par conséquent, nécessaire d'éclaircir leurs sens et leur emploi à partir des contextes offerts par le corpus.

\subsection{Les adjectifs dans les lexies spécialisées}

Dans cette section, nous passons à l'examen des adjectifs dans les lexies spécialisées ayant ENERGIE comme substantif. Après avoir délimité le vocable ENERGIE, le sens de chaque adjectif est approfondi dans les syntagmes nominaux; en outre, les relations existant entre les lexies spécialisées mêmes sont décrites par le biais de la fonction lexicale Syn. Rappelons que l'intérêt de cette approche réside dans l'identification de toute la gamme de liens lexicaux à partir d'une lexie spécialisée donnée (L'Homme $2012: 35)$.

Pour notre finalité, nous ne traiterons pas le vocable ENERGIE comme polysémique, mais nous nous focaliserons sur l'acception relevée du contexte et, plus précisement, de celui d'énergie renouvelable marine et des autres lexies spécialisées appartenant à cette classe conceptuelle. Parmi les paraphrases issues du TLFi pour ENERGIE, dont nous mentionnons 'principe d'action qui rend une personne apte à agir ou dont se trouve animée une chose pour agir sur la nature' et 'capacité d'un corps ou d'un système à produire du travail mécanique ou son équivalent', seule la deuxième, au sens de 'capacité' ou 'propriété', nous semble mieux circonscrire l'aire sémantique de l'unité lexicale dans les textes spécialisés de notre corpus. Pour le même terme, le DicoEnviro précise que l'énergie est produite par une source et qu'elle est utilisée par l'agent-homme. Sur la base des sources, les différents types d'énergie sont évoqués dans la section consacrée aux liens lexicaux : énergie de la biomasse, énergie éolienne, énergie géothermique, énergie hydraulique, énergie marémotrice, énergie marine, énergie solaire, bioénergie. Dans cette liste nous relevons trois lexies spécialisées qui font partie de notre analyse, énergie hydraulique, énergie marémotrice, énergie marine.

La lexie spécialisée énergie marine, attestée au singulier et au pluriel, est la plus fréquente dans le corpus : elle y figure 104 fois. Des trois définitions de marin illustrées par le TLFi, nous ne retenons que la première 'de mer, de la mer', de sorte que la lexie spécialisée énergie marine pourrait être paraphrasée comme "énergie de la mer' ${ }^{12}$. Cependant, une telle reformulation ne nous semble pas suffisante pour une compréhension exhaustive de la lexie. L'observation des contextes dans lesquels elle est employée peut offrir quelques points de repère utiles :

1) Cependant, avec des durées annuelles de fonctionnement en équivalent pleine puissance comprises entre 3000 et 7000 heures, voire 8000 heures (une année compte 8760 heures), les énergies marines ${ }^{13}$ pourront occuper une place de choix dans le mix énergétique, aux côtés d'autres énergies renouvelables, pour lisser la variabilité des productions.

2) La mission considère que le régime juridique actuel applicable en mer n'est pas assez favorable à un essor rapide des énergies marines.

3) La Grande Bretagne, et plus particulièrement l'Ecosse , est le chef de file dans le domaine des énergies marines, affichant à la fois une volonté de construire une chaîne d'approvisionnement locale et d'atteindre ses objectifs ambitieux de développement des énergies renouvelables.

4) Pour les opérations de montage et de maintenance des installations des énergies marines et, afin de limiter les opérations en mer, un maximum d'opérations d'assemblage et de logistique doivent être faites à terre, dans les ports à quai, ce qui implique des moyens de levage importants, des longueurs de quais et des zones logistiques et de stockage conséquentes. 
5) Pour les autres technologies d'énergies marines (éolien flottant, hydroliennes, énergie houlomotrice et énergie thermique des mers), la transposition immédiate n'est pas possible.

6) En 2007, l'ADEME, avec les Ministères concernés, le Secrétariat Général de la Mer et l'Ifremer, a développé un outil d'analyse stratégique, destiné aux services de l'État, pour déterminer les « zones pertinentes en faveur du développement de futurs parcs de production électrique en mer (d'origine renouvelable) 》 et s'est intéressée à plusieurs sources d'énergies marines comme l'éolien en mer, le houlomoteur ou encore l'hydrolien.

7) Les énergies marines concernées par cette AMI sont : l'hydrolien marin, l'houlomoteur, l'éolien flottant, l'énergie thermique marine.

L'exemple (1), qui donne des chiffres en termes de puissance et où l'on aborde le sujet de la production énergétique, suggère un sens de causalité inclus dans la lexie spécialisée, où marin acquiert le sens de 'qui est produit de la mer, à partir de la mer'. En revanche, les exemples (2), (4) et (6) semblent exprimer une composante locative (« le régime juridique actuel applicable en mer » $(2)$, « limiter les opérations en mer» $(4)$, « production électrique en mer» (6)), de sorte que marin signifie 'en mer, produit en mer' et que la lexie spécialisée énergie marine équivaut à 'énergie produite en mer'. De surcroît, dans les exemples (5), (6) et (7), il est possible de discerner les relations que la lexie spécialisée énergie marine entretient avec d'autres lexies spécialisées du domaine. Plus précisément, dans ces contextes (par exemple, «plusieurs sources d'énergies marines comme l'éolien en mer, le houlomoteur ou encore l'hydrolien ») elle fonctionne comme l'hyperonyme des lexies suivantes : énergie houlomotrice, énergie thermique des mers, énergie thermique marine, éolien flottant, éolien en mer, houlomoteur, hydrolien, hydroliennes. Cette relation peut être formalisée par la fonction lexicale suivante :

Syn (énergie marine) = énergie houlomotrice, énergie thermique des mers, énergie thermique marine, éolien flottant, éolien en mer, houlomoteur, hydrolien

Si nous ne considérons que les lexies spécialisées constituées du substantif énergie et de l'adjectif, l'adjectif marin pourrait être représenté en tant qu'hyperonyme par rapport aux autres :

$$
\begin{aligned}
& \operatorname{Syn}_{\supset}(\text { marin })=\text { houlomoteur, thermique des mers, thermique } \\
& \text { marin }
\end{aligned}
$$

Nous passons maintenant à l'examen de la deuxième lexie spécialisée énergies marines renouvelables, qui n'est pas attestée dans les trois dictionnaires consultés. Nous rappelons, pourtant, que les énergies renouvelables (DicoE) :

[...] sont essentiellement tirées des éléments (terre, eau, air et feu) et du soleil. On désigne aujourd'hui par énergies renouvelables un ensemble de filières diversifiées dont la mise en œuvre n'entraîne en aucune façon l'extinction de la ressource initiale.

Nous considérons le sens de la lexie spécialisée énergies marines renouvelables comme résultant de la composition des sens énergie + marine + renouvelable. Au cœur du syntagme se situe l'adjectif renouvelable, dérivé du verbe renouveler dont la structure prédicative ' $\mathrm{X}$ renouvelle $\mathrm{Y}$ ' a été évoquée dans la section 3.1. Le vocable ENERGIE constitue le $\mathrm{S}_{2}$ de renouveler et l'adjectif marin, se référant à l'eau, peut être décrit de manière double comme $S_{\text {instr }}$ ou $S_{\text {loc }}$. Les relations entre les trois éléments de la lexie peuvent être modélisées par trois fonctions lexicales distinctes dont la constante est renouveler: 


$$
\begin{aligned}
& \mathrm{S}_{2}(\text { renouveler })=\text { énergie } \\
& \mathrm{S}_{\text {instr }}(\text { renouveler })=\text { eau } \\
& \mathrm{S}_{\text {loc }}(\text { renouveler })=\text { eau }
\end{aligned}
$$

Nous pouvons observer l'emploi de la lexie spécialisée dans quelques contextes tirés du corpus :

8) Tout d'abord les énergies marines renouvelables « proprement dites », c'est-àdire les énergies dont le vecteur est l'eau des océans.

9) L'essor en France des énergies marines renouvelables (hors éolien posé pour lequel deux appels d'offres ont été lancés ou sont en préparation), suppose absolument de passer par la phase d'implantation de fermes-pilotes, afin de tester et de valider, sous forme de petits parcs de production d'électricité, les nouvelles technologies d'énergies marines, et cela dans des conditions réelles d'exploitation.

10) L'AMI a pour objectif d'accompagner des innovations, briques technologiques critiques et démonstrateurs permettant de consolider la filière énergies marines renouvelables (EMR).

11) [...] les perspectives de développement des énergies marines renouvelables (EMR) les plus matures (hors l'éolien offshore posé qui a déjà fait l'objet d'appels d'offres spécifiques lancés en 2012 et prévus en 2013 ) et des filières industrielles correspondantes : énergie hydrolienne, éolien offshore flottant, énergie houlomotrice et marémotrice, énergie thermique des mers, climatisation par eau de mer (SWAC) et énergie osmotique .

12) Autres éléments de réflexion et de proposition : la mise en place d'une gouvernance intégrant les associations de protection de l'environnement doit accompagner le développement des EMR, et la fiscalité liée aux énergies marines doit servir la protection de l'environnement.

L'exemple (8) précise que ce type d'énergie est réalisé grâce à l'élément naturel de l'eau, qui en est le vecteur. Cette propriété correspond à la particularité de la lexie spécialisée énergie marine que nous avons déjà commentée : les deux lexies spécialisées énergie marine et énergies marines renouvelables seraient-elles des synonymes absolus ? Les exemples du corpus semblent confirmer cette hypothèse : dans l'extrait (9), on a recours alternativement aux deux formes comme équivalentes; dans l'exemple (11), la liste des différents types d'énergies marines renouvelables correspond presque totalement à la liste des hyponymes d'énergie marine: nous retrouvons les lexies spécialisées énergie hydrolienne, énergie houlomotrice, marémotrice et énergie thermique des mers. En outre, comme nous l'avons observé plus haut, les deux lexies énergie marine et énergies marines renouvelables peuvent être reformulées de la même manière par une paraphrase telle que énergie qui peut être produite de l'élément naturel de l'eau'. L'ellipse de l'adjectif renouvelables dans certains contextes pourrait donc montrer sa rédondance, étant donné que le sémantisme de marin 'produit par la mer, ayant origine dans la mer' présuppose le sens de renouvelable 'produit par un élément naturel, ayant origine dans un élément naturel'. Les exemples (10) et (12) montrent une autre variation de la lexie énergies marines renouvelables, à savoir l'abréviation $E M R$. Ce phénomène peut être décrit par la fonction lexicale suivante :

$$
\text { Syn (énergies marines renouvelables) = énergies marines, EMR }
$$

En admettant cette relation de synonymie absolue, il est possible d'affirmer que la lexie spécialisée énergie marine accueille la même structure actancielle que celle d'énergies marines renouvelables. Bien qu'il soit implicite, l'élément prédicatif renouvelable doit être pris en considération. 
Comme nous venons de le voir, parmi les hyponymes d'énergies renouvelables marines figure énergie hydrolienne, dont 11 attestations sont repérables dans le corpus. À partir du sémantisme de l'adjectif identifié dans la section précédente, nous observons des contextes dans lesquels la lexie est utilisée :

13) L'énergie hydrolienne peut être assimilée à l'exploitation de puissantes rivières sous-marines ; la densité de l'eau est près de 850 fois supérieure à celle de l'air, et la production varie comme le cube de la vitesse du courant, ce qui explique que la ressource est localisée là où les courants de marée sont les plus forts.

14) Il n'existe pas encore (au niveau mondial) d'exploitation industrielle de l'énergie hydrolienne en mer, mais plusieurs fermes-pilotes de classe $10 \mathrm{MW}$ verront le jour à très court terme (2015), notamment au Royaume-Uni.

15) Un appel à manifestation d'intérêt « énergies marines » (AMI) pour développer les briques technologiques et les démonstrateurs de recherche d'énergie hydrolienne, houlomotrice, éolienne flottante et thermique des mers.

16) Énergie hydrolienne. Elle est produite par l'énergie des courants de marée qui sont concentrés dans certains endroits près des côtes.

Les contextes (13) et surtout (16) contribuent à mieux comprendre le sens de l'adjectif hydrolien, qui se réfère ici aux courants des marées, de sorte que la lexie spécialisée énergie hydrolienne acquiert le sens d'énergie dérivant des courants de marée, produite par les courants de marée'. Cependant, dans l'exemple (14), la lexie spécialisée énergie hydrolienne est suivie du complément de lieu " en mer», ce qui nous suggère, par analogie avec énergie renouvelables en mer, que ce type d'énergie pourrait relever non seulement de la mer, mais aussi d'autre ressources liées à l'élément de l'eau. Cette hypothèse est confirmée par les résultats de Termostat, qui recense hydrolien également comme le substantif des syntagmes hydrolien fluvial (7 attestations) et hydrolien maritime (2 attestations). De par cela, le sens de la lexie énergie hydrolienne correspond, plus en général, à 'énergie dérivant des courants, produite par les courants', ceux-ci pouvant se produire dans la mer ou dans le fleuve. Enfin, le contexte (15) explicite qu'énergie hydrolienne est une sous-classe d'énergies marines, aussi bien qu'énergie «houlomotrice, éolienne flottante et thermique des mers». Par conséquent, la lexie spécialisée peut être exprimée par ces fonctions lexicales :

$$
\begin{aligned}
& \mathrm{S}_{\text {instr }} \text { (énergie hydrolienne) = courant } \\
& \mathrm{Syn}_{c} \text { (énergie hydrolienne) = énergie marine, énergies marines renouvelables, EMR } \\
& \mathrm{Syn}_{\cap} \text { (énergie hydrolienne) = énergie houlomotrice, énergie éolienne flottante, } \\
& \text { énergie thermique des mers }
\end{aligned}
$$

En ce qui concerne la lexie spécialisée énergie houlomotrice, elle est définie dans le DicoE comme

l'énergie d'origine cinétique et potentielle liée au déplacement de la surface de la mer sous l'action de la houle. Une partie de cette énergie peut être récupérée grâce à différents dispositifs: flotteurs et radeaux oscillants, cloches à compression ou dépression.

Cette définition nous permet de mieux comprendre sa spécificité sémantique parmi les lexies cohyponymes et de préciser que cette énergie se produit «sous l'action de la houle». L'analyse des contextes aide à d'autres précisions :

17) Énergie houlomotrice: réduite par le mouvement des vagues (la houle), l'énergie houlomotrice est une forme concentrée de l'énergie du vent.

18) Énergie hydrolienne, éolien offshore flottant, énergie houlomotrice et marémotrice, énergie thermique des mers, climatisation par eau de mer (SWAC) et énergie osmotique. 
19) Pour les autres technologies d'énergies marines (éolien flottant, hydroliennes, énergie houlomotrice et énergie thermique des mers), la transposition immédiate n'est pas possible.

20) Aujourd'hui il y a plus de 100 projets à travers 15 pays, totalisant $1 \mathrm{GW}$ dont approximativement $60 \%$ en énergie houlomotrice et $40 \%$ d'énergie hydrolienne (données 2010).

21) L'énergie houlomotrice est en développement avec une floraison de technologies concurrentes.

L'exemple (17) suggère qu'à côté de 'houle', ou bien avant 'houle', $\mathrm{S}_{\text {instr }}$ serait 'vent'. D'autres cas (18) (19) (20) (21), en confirmant le lien de cohyponymie avec les autres lexies spécialisées par rapport à énergie marine, montrent une préférence en termes de cooccurrence avec marémotrice (18). L'intersection sémantique entre les deux adjectifs résulte du sens du radical commun motrice ainsi que du rapport de synonymie entre les substantifs houle et marée : elles renvoient, quelle que soit la source, au 'mouvement de la mer'. Ces relations peuvent être formalisées sous forme de fonction lexicale comme :

$$
\begin{aligned}
& \mathrm{S}_{\text {instr }} \text { (énergie houlomotrice) }=\text { houle } \\
& \mathrm{Syn}_{\cap}(\text { houlomotrice})=\text { marémotrice }
\end{aligned}
$$

Considérons maintenant la lexie énergie hydraulique, c'est-à-dire :

l'énergie qui provient de la force des eaux (cours d'eau et océans). Les centrales hydroélectriques ou usines marémotrices transforment l'énergie de la gravité de l'eau en énergie électrique grâce au courant de l'eau passant dans les turbines.

Cette description fournie par le DicoE souligne que son aire sémantique concerne la 'force des eaux' associée au 'mouvement du courant de l'eau' et se rapporte à n'importe quelle source, soit cours d'eau ou océans. Les contextes explicitent le sémantisme de la lexie :

22. L'arrêté tarifaire du 1er mars 2007 fixant les conditions d'achat de l'électricité produite par les installations utilisant l'énergie hydraulique des lacs, cours d'eau et mers prévoit, pour les énergies marines renouvelables (systèmes houlomoteurs, marémoteurs ou hydrocinétiques), un tarif d'achat de 163 pendant 20 ans.

23. La recherche et la définition d'un régime juridique cohérent et simplifié serait de nature à faciliter la valorisation et l'exploitation des ressources énergétiques marines, comme cela est déjà le cas (du point de vue juridique) pour l'énergie marémotrice, qui figure explicitement au titre V du Code de l'Énergie consacré à l'exploitation de l'hydroélectricité (installations utilisant l'énergie hydraulique).

24. L'énergie hydraulique récupérable atteint $40.10 \mathrm{kWh}$ et la valeur techniquement exploitable vaut, 12 selon les estimations, entre 15 et $20.10 \mathrm{kWh}$.

Dans (22), la lexie est suivie de la préposition « des » et de trois substantifs, « lac », « cours d'eau » et «mer » qui expriment une composante locative. Cela nous amène à considérer un autre type de fonction lexicale de type locatif (Loc), à savoir une préposition ou expression prépositive exprimant le sens 'se trouvant dans' (spatialement ou temporellement) (Mel'cuk, Clas, Polguère, 1995) :

$$
\begin{aligned}
& \mathrm{S}_{\text {instr }}(\text { énergie hydraulique })=\text { courant } \\
& \mathrm{S}_{\text {loc }}(\text { énergie hydraulique })=\operatorname{des} \text { [cours d'eau ; lacs ; mers] }
\end{aligned}
$$

Examinons, ensuite, le cas d'énergie osmotique pour constater, de même, que les informations issues de la langue générale à partir de l'adjectif osmotique ne sont pas suffisantes pour attester et circonscrire le positionnement sémantique de la lexie à l'intérieur de la classe des énergies marines renouvelables. À l'aide des informations contenues dans le DicoE, nous comprenons que, dans ce cas, 


\begin{abstract}
Une centrale osmotique génère de l'énergie par l'exploitation de l'énergie libérée par le mélange d'eau douce et d'eau de mer. L'énergie osmotique est une source d'énergie renouvelable et sans émissions de $\mathrm{CO} 2$. [...] Il en effet possible d'extraire de l'énergie en s'appuyant sur le phénomène d'osmose au voisinage des estuaires, des zones où l'eau douce entre en contact avec l'eau salée.
\end{abstract}

L'examen du corpus ne nous permet de retracer aucun lien paradigmatique particulier, à part celui d'hyponymie - formellement non motivé - par rapport à énergies marines renouvelables, comme dans (25) et (26);

25. Dans le cadre de la feuille de route pour la transition énergétique, adoptée par le gouvernement lors de la Conférence environnementale en septembre 2012, il a été demandé au CGEDD et au CGIET d'étudier les perspectives de développement des énergies marines renouvelables (EMR) les plus matures (hors l'éolien offshore posé qui a déjà fait l'objet d'appels d'offres spécifiques lancés en 2012 et prévus en 2013) et des filières industrielles correspondantes : énergie hydrolienne, éolien offshore flottant, énergie houlomotrice et marémotrice, énergie thermique des mers, climatisation par eau de mer (SWAC) et énergie osmotique.

26. En revanche, l'énergie osmotique n'est pas encore mature et nécessite une nouvelle phase de R et D. Enfin, l'énergie marémotrice, malgré un fort potentiel énergétique, se heurte à des difficultés d'intégration dans l'environnement (fermeture d'estuaires) ne permettant pas de projets réalistes en France.

\title{
$\mathrm{Syn}_{\supset}($ énergies marines renouvelables $)=$ énergie osmotique
}

Concluons par l'examen de la dernière lexie spécialisée, énergie marémotrice, que le DicoE paraphrase comme 'l'énergie provenant des phénomènes des marées', en précisant que ce procédé consiste à utiliser la puissance des marées pour produire de l'électricité ${ }^{14}$. Les contextes confirment cette définition et expliquent le mode d'exploitation de ce type d'énergie (27) (28): il en résulte que $\mathrm{S}_{\text {instr }}$ est 'marée'. Quant aux liens lexicaux, nous rappelons le rapport d'intersection sémantique avec la lexie énergie houlomotrice, qui se fonde sur une parenté morphologique entre les deux formes.

27. L'énergie marémotrice résulte de l'exploitation de l'énergie potentielle de la marée en utilisant les différences de niveau entre haute et basse mer.

28. L'énergie marémotrice désigne l'énergie provenant des phénomènes des marées. Ce procédé consiste à utiliser la puissance des marées pour produire de l'électricité.

$$
\mathrm{S}_{\text {instr }}(\text { (énergie marémotrice) }=\text { marée }
$$

$\mathrm{Syn}_{\cap}($ énergie marémotrice $)=$ énergie houlomotrice

\section{Conclusion}

Si les hyperonymes énergie marine/énergies marines renouvelables présentent une structure actancielle prédicative, l'analyse sémantique des autres adjectifs employés dans les lexies spécialisées semble confirmer notre hypothèse de départ: les adjectifs contribuent à discriminer les différentes classes d'énergies marines renouvelables sur la base de la source à travers laquelle ou dans laquelle est produite l'énergie. Bien qu'il existe un trait sémantique commun à tous les adjectifs considérés, à savoir l'origine dans l'élément aquatique, celui-ci est décliné dans ses différentes facettes: la mer (marin); le courant, marin ou fluvial (hydrolien); la houle (houlomoteur); la force ou le courant des eaux (hydraulique); le phénomène de l'osmose (osmotique); la marée (marémoteur). 
Compte tenu tant de la polysémie de certains adjectifs (nous pensons à marin) que de notre intérêt pour un domaine spécifique, se pose la nécessité d'examiner ces adjectifs également par rapport au vocable ENERGIE, c'est-à-dire dans les lexies spécialisées dont énergie constitue le nom tête. De par cela, il est possible de préciser le sens de chaque adjectif en éliminant, dans la mesure du possible, toute ambiguïté : dans les lexies spécialisées synonymiques énergie marine et énergies marines renouvelables, marin signifie 'qui est produit à partir de la mer, en mer' ; l'adjectif renouvelables, correspondant à 'qui peut être produit à nouveau d'un élément naturel' est parfois omis car jugé comme redondant. À partir des paraphrases données pour chaque lexie spécialisée, nous pouvons constater qu'énergie osmotique et énergie marémotrice ont un sens spécifique et un statut assez clair par rapport aux autres de la même classe ; en revanche, la différence entre énergie hydrolienne, énergie hydraulique et énergie houlomotrice - du fait du renvoi au même type d'instrument, 'le courant' - n'est pas saisissable à ce niveau d'analyse et demande d'autres recherches en contexte. Si ces exemples prouvent que «dans les dictionnaires de spécialité la plupart des termes complexes ont un signifié compositionnel ${ }^{15}$ (L'Homme $2007: 4$ ), car les sens originaires des adjectifs sont confirmés lorsqu'ils cooccurrent avec énergie, le statut sémantique de l'adjectif marin, par contre, montre un écart plus significatif. Les données de notre échantillon signalent une extension métaphorique de l'adjectif, de sorte que l'étiquette « énergie marine » candidate à la désignation de toute la gamme des énergies renouvelables qui exploitent la force des eaux, et non seulement celles qui se produisent « en mer». La modélisation par fonctions lexicales contribue simultanément à une meilleure compréhension de la structure conceptuelle du domaine et des relations entre lexies. Énergie marine / énergies marines renouvelables sont les hyperonymes, dont la structure prédicative du verbe renouveler, même lorsqu'il est omis, est transféré aux cohyponymes. Toutefois, la synonymie, totale ou partielle, de certains adjectifs (marin/marines renouvelables; hydrolien/hydraulique) pourrait témoigner d'un flou terminologique, qui n'est pas étonnant dans un secteur dont les technologies sont l'objet d'un développement récent.

\section{Références}

Assadi, S., Bourigault, D. (1995). Classification d'adjectifs extraits d'un corpus pour l'aide à la modélisation de connaissances. Troisièmes journées internationales d'analyse des données textuelles, pp. 313-320.

Bartning, I. (1980). Remarques sur la syntaxe et la sémantique des pseudo-adjectifs dénominaux en français. Stockholm : Almqvist \& Wiksell International.

Bosredon, A. (1988). Un adjectif de trop : l'adjectif de relation. L'information grammaticale, 37, pp. 3-7.

Bourigault, D., Slodzian, M. (1999). Pour une terminologie textuelle. Terminologies Nouvelles, Spécial TIA 19, pp. 29-32.

Bourigault, D. (1993). Analyse syntaxique locale pour le repérage de termes complexes dans un texte. Traitement automatique des langues, 34(2), pp.105-117.

Cabré, T., Condamines, A., Ibekwe-SanJuan, F. (2005). Introduction: Application-driven terminology engineering. Terminology, 11(1), pp. 1-19.

Cabré, T. (2003). Theories of terminology: Their description, prescription and explanation. Terminology, 9(2), pp. 163-199.

Calberg-Challot, M., Candel, D., Bourigault, D., Dumont, X., Humbley, J., Joseph, J. (2008). Une analyse méthodique pour l'extraction terminologique dans le domaine du nucléaire. Terminology, 14(2), pp. 183-203.

Daille, B. (2001). Qualitative Term Extraction: Identifying relational adjectives. Bourigault, D., Jacquemin, C., L'Homme, M.-C. (eds.). Recent Advances in Computational Terminology, Amsterdam/Philadelphia : John Benjamins, pp. 149-166.

DiCoEnviro, Le dictionnaire fondamental de l'environnement. Observatoire de linguistique Sens-Text, Université de Montréal. http://olst.ling.umontreal.ca/cgi-bin/dicoenviro/search_enviro.cgi (dernière consultation : 20.02.2016).

Dictionnaire Environnement http://www.dictionnaire-environnement.com/ (dernière consultation : 30.11.2015).

Dixon, R.M.W. (1982). Where Have All the Adjectives Gone?. Dixon, R.M.W., Where Have All the Adjectives Gone? And other Essays in Semantics and Syntax. Berlin/New-York/Amsterdam: Mouton, pp. 1-62.

Drouin, P. (2003). Term extraction using non-technical corpora as a point of leverage. Terminology, 9(1), pp. 99-117. 
Gentilhomme, Y. (1994). L'éclatement du signifié dans les discours technoscientifiques. Cahiers de lexicologie. 64(1), pp. 5-35.

Frassi, P. (2014). La définition lexicographique des dérivés sémantiques adjectivaux. SHS Web of Conferences, 8, pp. 741-758.

Giaufret, A. (2013). La terminologie des énergies renouvelables dans les bases de données terminologiques: les exemples de IATE et du GDT. Ligas P., Tallarico G., (dir), Lexique, lexiques, Verona : Qui Edit, pp. 51-72.

Giaufret, A., Rossi M. (dir.) (2012). La terminologia delle energie rinnovabili tra testi e repertori: variazione, standardizzazione, armonizzazione. Genova : GUP.

Goes, J. (1993). À la recherche d'une définition de l'adjectif. L'information grammaticale, 58, pp. 11-13.

Hladik, J. (2011). Les énergies renouvelables aujourd'hui et demain. Paris : Ellipses.

L'Homme, M.-C. (2012). Using ECL (Explanatory Combinatorial Lexicology) to discover the lexical structure of specialized subject fields. Apresjan, J. et al. (eds.). Words, Meanings and other Interesting Things. A Festschrift $n$ Honour of the 80th Anniversary of Professor Igor Alexandrovic Mel'cuk, Moscow : RCK, pp. 378-390.

L'Homme, M.-C. (2007). Using Explanatory and Combinatorial Lexicology to Describe Terms. Wanner, L. (éd.) Selected Lexical and Grammatical Topics in the Meaning-Text Theory. In Honour of Igor Mel'cuk. Amsterdam/Philadelphia : John Benjamins.

L'Homme, M.-C. (2004). Adjectifs dérivés sémantiques (ADS) dans la structuration des terminologies. Actes de Terminologie, ontologie et représentations des connaissances. (Université Jean Moulin Lyon 3, 22-23 janvier 2004).

L'Homme, M.-C. (2002a). Nouvelles technologies et recherche terminologique. Techniques d'extraction des données terminologiques et leur impact sur le travail du terminographe. L'impact des nouvelles technologies sur la gestion terminologique. http://olst.ling.umontreal.ca/pdf/textHomme.pdf (dernière consultation : 8.03.2016).

L'Homme, M.C. (2002b). Fonctions lexicales pour représenter les relations entre termes. Traitement automatique des langues (TAL), 43(1), pp. 19-41.

L'Homme, M.-C., Benali, L., Bertrand, C., Lauduique, P. (1996). Definition of an Evaluation Grid for TermExtraction Software. Terminology, 3(2), pp. 291-312.

Maniez, F. (2009). L'adjectif dénominal en langue de spécialité : étude du domaine de la médecine. Revue française de linguistique appliquée, 2, vol. XIV, pp. 117-130.

Marcel, É. (2000). Quelques modalités d'application de la fonction lexicale gener en botanique. Cahiers de lexicologie, 76(1), pp. 91-110.

Mel'čuk, I. (2004a). Actants in semantics and syntax I: Actants in semantics. Linguistics, 42(1), pp. 1-66.

Mel'čuk, I. (2004b). Actants in semantics and syntax II: Actants in syntax. Linguistics, 42(2), pp. 247-291.

Mel'čuk, I. (1996). Lexical Functions: A Tool for the Description of Lexical Relations in the Lexicon. Wanner, L. (ed.) Lexical Functions in Lexicography and Natural Language Processing. Amsterdam/Philadelphia: Benjamins, pp. 37-102.

Mel'čuk, I. A., Clas, A., Polguère, A. (1995). Introduction à la lexicologie explicative et combinatoire. Louvain-laNeuve : Duculot / Aupelf -UREF.

Mel'čuk, I. (1974). Opyt teorii lingvistic]eskix modelej "Smysl $<>$ Tekst". Semantika, Sintaksis [Esquisse d'une théorie des modèles linguistiques du type Sens $<>$ Texte. La sémantique, la syntaxe]. Moskva : Nauka.

Meyer, I., Mackintosh, K., Barrière, C., Morgan, T. (1999). Conceptual sampling for terminological corpus analysis. Sandrini, P. (ed.). Proceedings of TKE '99: Fifth International Congress on Terminology and Knowledge Engineering, 23-27 août 1999. Innsbruck, Austria : TermNet, pp. 256-267.

Meyer, I., Mackintosh, K. (1996). The corpus from a terminographer's viewpoint. International Journal of Corpus Linguistics, 1(2), pp. 257-285.

Noailly, M. (1999). L'adjectif en français. Paris : Ophrys.

Riegel, M. (1993). Grammaire et référence : à propos du statut sémantique de l'adjetcif qualificatif. L'information grammaticale, 58 , pp. 5-10.

Rossi, M. (2014). Les énergies renouvelables entre description lexicographique et traitement terminologique : la diffusion des termes des énergies renouvelables dans les dictionnaires de langue générale. Rivista Italiana di Linguistica Applicata, 3, pp.77-98.

Trésor de la langue française informatisé. atilf.atilf.fr (dernière consultation : 30.11.2015).

Van Campenhoudt, M. (1995). Les relations hiérarchiques. Terminology, 3(2), pp. 53-83.

Wierzbicka, A. (1988). What's in a Noun? (Or: How do Nouns Differ in Meaning from Adjectives). Wierzbicka, A. The Semantics of Grammar. Amsterdam : J. Benjamins, pp. 463-497.

Zanola M.T. (dir.) (2012), Costruire un glossario : la terminologia dei sistemi fotovoltaici. Milano : Vita \& Pensiero.

Zanola, M.T. (2008). Énergies renouvelables et protection de l'environnement : des problèmes de terminologie juridique. Actes Realiter 2008. http://www.realiter.net/hp/1084-2/1128-2 (dernière consultation : 26.11.2015).

Žolkovskij, A., Mel'čuk, I. (1967). O semantičeskom sinteze [Sur la synthèse sémantique]. Problemy kibernetiki, 19, pp. 177-238. 
1 Ministère de l'Écologie, du Développement durable et de l'Énergie, Énergies renouvelables en mer, $\mathrm{http}: / / \mathrm{www}$.developpement-durable.gouv.fr/-Energies-marines-renouvelables,4757-.html (dernière consultation : 26.11.2015).

${ }^{2}$ Ministère de l'Écologie, du Développement durable et de l’Énergie, Faire de la France un leader mondial des énergies marines, http://www.developpement-durable.gouv.fr/Faire-de-la-France-un-leader,31646.html (dernière consultation : 26.11.2015).

${ }^{3}$ P. Drouin, http://termostat.ling.umontreal.ca/. Voir aussi Drouin, P. (2003). Term extraction using non-technical corpora as a point of leverage. Terminology, 9 (1), pp. 99-117.

${ }^{4}$ Nous traitons ensemble les formes du singulier énergie marine et du pluriel énergies marines, qui sont pourtant distingués dans les résultats élaborés par le logiciel, la première ayant une fréquence de 100, la seconde de 4.

${ }^{5}$ Nous signalons également les cas de la fréquence comprise égale ou inférieure à $1 \%$, mais supérieure à $0 \%$ : Nom Préposition Nom Préposition Nom Adjectif $=36$ (1\%); Nom Adjectif Préposition Nom Adjectif= 31 (1\%); Nom Préposition Nom Adjectif Adjectif $=28(1 \%)$; Nom PPAdj Préposition Nom= $15(0.4 \%)$; Nom PPAdj Adjectif $=14$ $(0.3 \%)$; Nom Adjectif Préposition Nom Préposition Nom= 10 (0.2\%); Nom Adjectif Adjectif Adjectif $=10(0.2$ $\%)$; Nom Préposition Nom Préposition Nom Préposition Nom=9 (0.2\%); Nom Adjectif Adjectif PPAdj= $9(0.2$ $\%)$; Nom Préposition Nom Adjectif Préposition Nom= 6 (0.1\%); Nom PPAdj PPAdj= $6(0.1 \%)$; Nom Adjectif Adjectif Préposition Nom= $5(0.1 \%)$; Nom Préposition Nom Adjectif Adjectif Adjectif $=4(0.1 \%)$; Nom PPAdj Préposition Nom Adjectif $=3(0.1 \%)$; Nom Adjectif PPAdj Adjectif $=3(0.1 \%)$.

${ }^{6}$ Trésor de la langue française informatisé, atilf.atilf.fr (dernière consultation : 30.11.2015).

${ }^{7}$ Le dictionnaire fondamental de l'environnement, http://olst.ling.umontreal.ca/cgi-bin/dicoenviro/search_enviro.cgi (dernière consultation : 20.02.2016).

${ }^{8}$ Dictionnaire Environnement, http://www.dictionnaire-environnement.com/ (dernière consultation : 30.11.2015).

9 «[...] terminology is much more comfortable with non-predicative units (or units considered as being nonpredicative), and much less with predicative units such as verbs and adjectives ».

${ }^{10}$ Voir aussi la définition de l'adjectif houleux 'qui est agité par le mouvement ondulatoire de la houle' (TLFi).

${ }^{11}$ D'autres informations sont fournies sur les liens lexicaux d'hydraulique, notamment avec les adjectifs marin, solaire et thermique.

${ }^{12}$ L'entrée énergie marine n'est pas enregistrée dans le Dictionnaire Environnement.

${ }^{13}$ C'est nous qui soulignons.

${ }^{14}$ Le DicoE ajoute qu'en France, EDF exploite l'usine marémotrice de La Rance en Bretagne, la seule installation à ce jour capable de transformer de façon industrielle l'énergie des marées en électricité.

15 « In specialized dictionaries, a very large portion of complex nouns has a compositional meaning » (c'est nous qui traduisons). 